\title{
Error estimates for a mixed finite element discretization of some degenerate parabolic equations
}

\author{
Florin A. Radu • Iuliu Sorin Pop • Peter Knabner
}

Received: 30 January 2007 / Revised: 17 December 2007 / Published online: 15 February 2008

(C) Springer-Verlag 2008

\begin{abstract}
We consider a numerical scheme for a class of degenerate parabolic equations, including both slow and fast diffusion cases. A particular example in this sense is the Richards equation modeling the flow in porous media. The numerical scheme is based on the mixed finite element method (MFEM) in space, and is of one step implicit in time. The lowest order Raviart-Thomas elements are used. Here we extend the results in Radu et al. (SIAM J Numer Anal 42:1452-1478, 2004), Schneid et al. (Numer Math 98:353-370, 2004) to a more general framework, by allowing for both types of degeneracies. We derive error estimates in terms of the discretization parameters and show the convergence of the scheme. The features of the MFEM, especially of the lowest order Raviart-Thomas elements, are now fully exploited in the proof of the convergence. The paper is concluded by numerical examples.
\end{abstract}

\footnotetext{
F. A. Radu

Max-Planck Institute for Mathematics in the Sciences, Inselstr. 22, 04103 Leipzig, Germany

F. A. Radu $(\bowtie)$

UFZ, Helmholtz Center for Environmental Research, Permoserstr. 15, 04318 Leipzig, Germany

e-mail: florin.radu@ufz.de

I. S. Pop

Department of Mathematics and Computer Science, Eindhoven University of Technology, P.O. Box 513, 5600 MB, Eindhoven, The Netherlands e-mail: I.Pop@tue.nl

P. Knabner

Institute of Applied Mathematics, University Erlangen-Nürnberg,

Martensstr. 3, 91058 Erlangen, Germany

e-mail: knabner@am.uni-erlangen.de
} 
Mathematics Subject Classification (2000)

$65 \mathrm{M} 12 \cdot 65 \mathrm{M} 15 \cdot 65 \mathrm{M} 60 \cdot 35 \mathrm{~K} 65 \cdot$ $35 \mathrm{~K} 55 \cdot 76 \mathrm{~S} 05$

\section{Introduction}

In this paper we analyze a mixed finite element scheme for the nonlinear, possibly degenerate, parabolic equation

$$
\partial_{t} b(u)-\nabla \cdot\left(\nabla u+k(b(u)) \mathbf{e}_{z}\right)=0,
$$

where $\mathbf{e}_{z}$ denotes the vertical unit vector. This paper continues the work in $[26,30]$, obtained for a Lipschitz continuous nonlinearity $b(\cdot)$. Here we give a simplified convergence proof in a more general context, by assuming that $b(\cdot)$ is only Hölder continuous. Therefore both types of degeneracy are allowed; to be specific, for some $\bar{u} \in \mathbb{R} \bigcup\{ \pm \infty\}$ the following situations may occur:

(a) $u \rightarrow \bar{u}$ implies $b^{\prime}(u) \rightarrow 0$ : the fast diffusion case,

(b) $u \rightarrow \bar{u}$ implies $b^{\prime}(u) \rightarrow \infty$ : the slow diffusion case.

In particular, the vanishing of $b^{\prime}(\cdot)$ may occur on intervals.

Many porous media models can be brought to the form in (1.1). In this sense we mention the equation

$$
\partial_{t} \Theta(\psi)-\nabla \cdot(K(\Theta(\psi)) \nabla(\psi+z))=0,
$$

which has been proposed by L.A Richards in 1930 to model the water flow in a porous medium (see e.g. [10]). In (1.2), $\psi$ denotes the pressure head, $\Theta$ the saturation reduced to the standard interval $[0,1], K$ stands for the hydraulic conductivity of the medium and $z$ for the height against the gravitational direction. Based on experimental results, different curves have been proposed for describing the dependency between $K, \Theta$ and $\psi$ (see e. g. [10]), yielding the nonlinear model (1.2). In this sense we mention the van Genuchten - Mualem framework, where

$$
\Theta(\psi)=\left(1+(c|\psi|)^{\frac{1}{1-m}}\right)^{-m}, \quad K(\Theta)=K_{s} \Theta^{\frac{1}{2}}\left[1-\left(1-\Theta^{\frac{1}{m}}\right)^{m}\right]^{2},
$$

whenever the flow is unsaturated $(\psi<0)$. Here $K_{s}>0, c>0$ and $m \in(0,1)$ are medium dependent parameters. For the fully saturated regime $(\psi \geq 0)$ we have $\Theta=1$ and $K=K_{s}$. Notice that in the present setting Richards' equation degenerates whenever $\psi$ goes to $-\infty$, implying that both $\Theta^{\prime}(\psi)$ and $K(\Theta(\psi))$ are approaching 0 , or in the fully saturated regime $(\psi \geq 0)$, when $\Theta^{\prime}(\psi)=0$. The regions of degeneracy depend on the saturation of the medium; therefore these regions are not known a-priori and may vary in time and space.

The Richards equation is written in the pressure based formulation. In this way all flow regimes up to the case of completely dry soils can be considered: unsaturated, partially saturated and fully (water) saturated. As indicated in [3], the 
Kirchhoff transformation

$$
\begin{aligned}
\mathcal{K}: \mathbb{R} & \longrightarrow \mathbb{R} \\
\psi & \longmapsto \int_{0}^{\psi} K(\Theta(s)) d s
\end{aligned}
$$

allows writing the model in the more regular unknown $u:=\mathcal{K}(\psi)$. Notice that the hydraulic conductivity $K$ can only vanish in the completely unsaturated case, when $\Theta=0$. Hence $K(\Theta(s))>0$ whenever $\Theta>0$, so the transformation is bijective. With

$$
\begin{aligned}
& b(u) \quad:=\Theta \circ \mathcal{K}^{-1}(u), \\
& k(b(u)):=K \circ \Theta \circ \mathcal{K}^{-1}(u),
\end{aligned}
$$

we can bring Eq. (1.2) to the form stated in (1.1). Further, for the nonlinearities in (1.3), $b^{\prime}(\cdot)$ vanishes in the fully saturated regime, when $u \geq 0$. In this case the Eq. (1.1) becomes elliptic and we have the fast diffusion case at $\bar{u}=0$. Moreover, in the above setting $u$ approaches a negative value $M$ as the soil is drying, which translates into $\psi \rightarrow-\infty$ in (1.2). In this case $b^{\prime}(\cdot)$ is blowing up. This corresponds to a vanishing diffusion in the original form (1.2), and the equation becomes hyperbolic or ordinary. Clearly, for this important situation, assuming the Lipschitz continuity of $b(\cdot)$ is too restrictive.

Another example is the porous medium equation (see [6])

$$
\partial_{t} v=\Delta v^{m}
$$

with $m \geq 1$. Here we seek for nonnegative solutions $v$; to be rigorous we have to extend the nonlinearity in the Laplacian by 0 for negative arguments. However, we restrict here to physically relevant cases, where the data is non-negative. In this case we can prove the maximum principle for $(1.6)$ (see, e.g. $[18,20]$ ), therefore the solution remains non-negative for all times and the extension of the nonlinearity plays no role. Taking $u=v^{m}$ and $b(u)=u^{1 / m}$, as well as $k \equiv 0$, we end up with the Eq. (1.1). In this case the degeneracy appears at $u=0$, for which $b^{\prime}(\cdot)$ becomes unbounded. Again we have a slow diffusion case at $\bar{u}=0$.

Finally, a third important equation in the class considered here models solute transport in porous media, involving equilibrium adsorption (see [7,13]):

$$
\partial_{t}(u+\Phi(u))-\nabla \cdot(\nabla u-\mathbf{Q} u)=0 .
$$

Depending on the specific situation, the sorption isotherm $\Phi$ may be non-Lipschitz, as appearing for example in the case of a Freundlich isotherm when $\Phi(u)=C u^{p}$ for some $p \in(0,1)$. Here $u$ denotes the concentration of a dissolved substance, whereas the water content, the bulk density, as well as the diffusion-dispersion coefficient are assumed constant and scaled to 1 . Furthermore, $\mathbf{Q}$ denotes the flux of the underlying flow field. In this case we encounter a slow diffusion degeneracy at $\bar{u}=0$. 
Having in mind the above examples, we are interested in solving equation (1.1) endowed with initial and boundary conditions

$$
\begin{aligned}
\partial_{t} b(u)-\nabla \cdot\left(\nabla u+k(b(u)) \mathbf{e}_{z}\right) & =0 \text { in }(0, T] \times \Omega, \\
u & =u_{I} \text { in } 0 \times \Omega, \\
u & =0 \text { on }(0, T] \times \Gamma .
\end{aligned}
$$

In the above problem $\Omega$ is a $d$-dimensional domain, where $d=1,2$ or 3 . Its boundary is denoted by $\Gamma$. Further, $T>0$ is a given finite time.

Throughout this paper we make use of the following assumptions:

(A1) $\Omega \subset \mathbb{R}^{d}$ is open, bounded and has a Lipschitz continuous boundary.

(A2) $b(\cdot) \in C^{0, \alpha}$ is nondecreasing and Hölder continuous: there exists an $\alpha \in(0,1]$ and $C_{b}>0$ so that $\left|b\left(u_{1}\right)-b\left(u_{2}\right)\right| \leq C_{b}\left|u_{1}-u_{2}\right|^{\alpha}$ for all $u_{1}, u_{2} \in \mathbb{R}$. For simplicity we assume $b(\cdot)$ continuously differentiable almost everywhere.

(A3) $k(b(\cdot))$ is continuous and bounded and satisfies for all $u_{1}, u_{2} \in \mathbb{R}$, $\left|k\left(b\left(u_{2}\right)\right)-k\left(b\left(u_{1}\right)\right)\right|^{2} \leq C_{k}\left(b\left(u_{2}\right)-b\left(u_{1}\right)\right)\left(u_{2}-u_{1}\right)$.

(A4) The initial data satisfies $u_{I} \in L^{2}(\Omega)$.

An additional assumption will be needed to obtain the error estimates in the fully discrete approximation. Since this involves the discretized fluxes, we do not state it here, but where required.

Remark 1.1 For the Richards equation in the Mualem - van Genuchten setting, the assumption (A2) holds with $\alpha=2 m /(3 m+2)$ (so $b(\cdot)$ is not Lipschitz!), whereas (A3) is satisfied whenever $m \in[2 / 3,1)$. For the porous medium equation (A2) is satisfied with $\alpha=1 / m$.

Remark 1.2 In (1.8) we have considered only a vertical convection. The results in this paper can be straightforwardly extended to the more general case, where the convection term is a vector satisfying (A3). Furthermore, $\Delta u$ may be replaced by a more general operator $\nabla \cdot \mathbf{D} \nabla u$, with $\mathbf{D}$ being a uniformly positive definite tensor. In this case, the proof is done by a slight modification of the Proposition 3.1.

Remark 1.3 For the ease of presentation we have only considered homogeneous Dirichlet boundary conditions. The results can be extended to more general ones, as well as for problems involving a reaction term satisfying a condition that is similar to (A3).

In this paper we analyze the order of convergence for a mixed finite element spatial discretization combined with an implicit Euler discretization in time for the Eq. (1.1). MFEM and its variants [11], together with cell centered finite volume methods like in [5], the Multipoint Flux Approximation Method (MPFA) [1] or combinations of MFEM and finite volume [17] belong to the class of locally mass conservative methods which recently have attracted much attention. In addition, the MFEM provides an accurate explicit flux approximation, a property that is of crucial importance if a fluid transport module like the Richards equation is coupled with a subsequent (multicomponent reactive) solute transport module by providing the underlying driving fluid 
flux. We mention $[4,9,26,30,35]$ for a mixed finite element discretization of (1.1). Specifically, the lowest order Raviart-Thomas finite elements are used, whereas the time discretization is achieved by an Euler implicit scheme. For the spatial discretization, optimal error estimates are obtained in $[4,30]$. For proving the convergence of the fully discrete scheme, the solution is assumed sufficiently regular. Similar results are obtained in [34] for an expanded MFEM, where three variables are considered explicitly: the pressure, its gradient and the flux. We also mention the combined finite volume - MFEM approach analyzed in [17].

There are many results on the convergence analysis of the conformal finite element discretization combined with a one step time discretization. Due to the low global regularity of the solutions of degenerate parabolic problems, in general only piecewise linear ansatz functions are considered. A similar situation appears for the MFEM. The convergence results for the scalar unknowns obtained by both conformal and mixed methods are comparable. While for conformal approaches also estimates that are pointwise in time are available, the mixed approach is providing valuable information on the approximation of the flux. This is due to the specific nature of the method. For the numerical analysis of conformal discretizations of the Richards equation in the pressure formulation we refer to $[16,21]$ and in the saturation based formulation to [23], where both type of degeneracy are allowed but the results do not apply to the fully saturated flow regime. We mention [7] for the equilibrium sorption transport problem, as well as $[14,24]$ for the porous medium equation.

In the present paper we prove the convergence of the lowest order Raviart-Thomas mixed finite element, Euler implicit discretization in a general framework. It continues the work in [26], where the ideas in $[4,30]$ are combined with the techniques for degenerate parabolic equations that are developed in [21]. The convergence order estimates in [26] are obtained for a Lipschitz continuous nonlinearity $b(\cdot)$. An essential point of the proof is the equivalence between the mixed and conformal formulations, for both the continuous and the time discrete problems.

Here we only assume the Hölder continuity of $b(\cdot)$. Therefore both degeneracies mentioned before are allowed. This applies in particular to the Richards equation (1.2) and extends the results presented in $[26,30]$. Furthermore, the error estimates are obtained here in a transparent manner. Though the equivalence to a conformal formulation remains valid in this general framework, it plays a secondary role in the proof. It is required only for the regularity of the solution. We make use of the possibility to have $L^{2}$ test functions in the mass conservation equation, which is a particular feature of the lowest order Raviart-Thomas finite elements. This leads to a simplified convergence proof for the semidiscrete scheme, avoiding the techniques involving the Green operator used in $[21,23,24,26]$. Notice also that in this work we do not assume $b(u) \in L^{\infty}\left(0, T ; L^{\infty}(\Omega)\right)$, as commonly done in the literature (see e. g. $[4,34])$. Another advantage of the present approach is that the convergence in the nondegenerate case, as well as in the fast diffusion case where $b(\cdot)$ is Lipschitz continuous, can be obtained directly as particular cases of the current results. We also mention that in many papers a regularized problem is considered as an intermediate step in obtaining the convergence results, where an adaption rule between the regularization and the discretization parameters is required. Here we avoid such a regularization step and make the result more transparent. 
The paper is organized as follows. In Section 2 the mixed continuous variational formulation is stated and the regularity of the solution is discussed. The error estimates for the time discrete scheme are obtained in the next section. The fully discrete scheme is considered in Section 4, where error estimates are derived in terms of the discretization parameters. In Section 5 we present some convergence tests and the conclusions.

\section{The mixed formulation}

In what follows we seek for weak solutions for the problem (1.8), written in the mixed form. We start with the weak conformal formulation, and investigate the equivalence between the mixed and conformal solutions. To define a solution in the weak sense, we make use of common notations in the functional analysis. By $\langle\cdot, \cdot\rangle$ we mean the inner product on $L^{2}(\Omega)$, or the duality pairing between $H_{0}^{1}(\Omega)$ and $H^{-1}(\Omega)$. Further, $\|\cdot\|,\|\cdot\|_{1}$ and $\|\cdot\|_{-1}$ stand for the norms in $L^{2}(\Omega), H^{1}(\Omega)$, respectively $H^{-1}(\Omega)$. The functions in $H$ (div; $\Omega$ ) are vector valued, having a $L^{2}$ divergence. By $C$ we mean a positive constant, not depending on the unknowns or the discretization parameters.

A weak, conformal solution of (1.8) solves the following problem:

Problem $P_{C}$. Find $u \in L^{2}\left(0, T ; H_{0}^{1}(\Omega)\right)$ such that $b(u) \in H^{1}\left(0, T ; H^{-1}(\Omega)\right)$, $u(0)=u_{I} \in L^{2}(\Omega)$, and

$$
\int_{0}^{T}\left\langle\partial_{t} b(u(t)), \varphi(t)\right\rangle+\left\langle\nabla u(t)+k(b(u(t))) \mathbf{e}_{z}, \nabla \varphi(t)\right\rangle d t=0
$$

for all $\varphi \in L^{2}\left(0, T ; H_{0}^{1}(\Omega)\right)$.

Existence, uniqueness and essential boundedness for a weak solution of (1.8) is studied in several papers (see for example [3,22] and the references therein). We also mention [32] for the analysis of an outflow problem in unsaturated media that is based on regularization. In particular, the following regularity is proven in [3]

$$
\begin{aligned}
b(u) & \in L^{\infty}\left(0, T ; L^{1}(\Omega)\right), \\
\mathbf{q}:=-\left(\nabla u+k(b(u)) \mathbf{e}_{z}\right) & \in L^{2}\left(0, T ;\left(L^{2}(\Omega)\right)^{d}\right) .
\end{aligned}
$$

Since $b(u) \in H^{1}\left(0, T ; H^{-1}(\Omega)\right)$ we have $b(u) \in C\left(0, T ; H^{-1}(\Omega)\right)$. Moreover, by the Hölder continuity of $b(\cdot)$, since $u \in L^{2}\left(0, T ; H_{0}^{1}(\Omega)\right)$ we have $(\alpha \in(0,1])$

$$
b(u) \in L^{2}\left(0, T ; L^{2 / \alpha}(\Omega)\right) \subseteq L^{2}\left(0, T ; L^{2}(\Omega)\right) .
$$

As stated in the introduction, our aim is to prove the convergence of a mixed finite element discretization of (1.1). Due to the degeneracy of this equation, its solution lacks regularity. In particular, $\partial_{t} b(u)$ is only in $L^{2}\left(0, T ; H^{-1}(\Omega)\right)$, so in the variational formulation of (1.1) the spatial regularity of the test functions should be $H^{1}$. However, 
the mixed formulation requires test functions that are only $L^{2}$ in space. To overcome this difficulty we follow [4] (see also [34]) and integrate (1.1) in time from 0 to any $t \in(0, T]$. With q defined in (2.3) this gives

$$
b(u(t))+\nabla \cdot \int_{0}^{t} \mathbf{q}(s) d s=b\left(u_{I}\right)
$$

for all $t$, but only in the sense of $H^{-1}$.

Further, since $b(\cdot)$ is Hölder continuous, by (A4) we have also $b\left(u_{I}\right) \in L^{2 / \alpha}(\Omega) \subseteq$ $L^{2}(\Omega)$. Using this as well as (2.4), from (1.1), (2.3) and (2.5) we conclude that

$$
\int_{0}^{t} \mathbf{q} d s \in X:=H^{1}\left(0, T ;\left(L^{2}(\Omega)\right)^{d}\right) \cap L^{2}(0, T ; H(\operatorname{div} ; \Omega)) .
$$

This yields

$$
\nabla \cdot \int_{0}^{t} \mathbf{q} d s \in L^{2}(\Omega)
$$

for almost every $t$.

To summarize, from (2.5) we have for all $\varphi \in H_{0}^{1}(\Omega)$ and $t \in(0, T]$

$$
\left\langle b(u(t), \varphi\rangle+\left\langle\nabla \cdot \int_{0}^{t} \mathbf{q}(s) d s, \varphi\right\rangle=\left\langle b\left(u_{I}\right), \varphi\right\rangle .\right.
$$

Moreover, $b(u(t))$ and $\nabla \cdot \int_{0}^{t} \mathbf{q}(s) d s$ are $L^{2}$ for almost every $t$. Since these are defined for all $t$, by density arguments we conclude that Eq. (2.7) holds for all $\varphi \in L^{2}(\Omega)$ and all $t$. In this way we can now define the mixed, time integrated variational form of (1.8):

Problem $P_{M}$. Find $(p, \mathbf{q}) \in L^{2}\left(0, T ; L^{2}(\Omega)\right) \times L^{2}\left(0, T ;\left(L^{2}(\Omega)\right)^{d}\right)$ such that $\int_{0}^{t} \mathbf{q}(s) d s \in X$, and

$$
\begin{array}{r}
\left\langle b(p(t))-b\left(p^{0}\right), w\right\rangle+\left\langle\nabla \cdot \int_{0}^{t} \mathbf{q}(s) d s, w\right\rangle=0, \\
\left\langle\int_{0}^{t} \mathbf{q}(s) d s, \mathbf{v}\right\rangle-\left\langle\int_{0}^{t} p(s) d s, \nabla \cdot \mathbf{v}\right\rangle+\left\langle\int_{0}^{t} k(b(p(s))) \mathbf{e}_{z} d s, \mathbf{v}\right\rangle=0,
\end{array}
$$

for all $t \in(0, T], w \in L^{2}(\Omega)$ and $\mathbf{v} \in H(\operatorname{div} ; \Omega)$, with $p(0)=u_{I} \in L^{2}(\Omega)$.

The problems $P_{C}$ and $P_{M}$ are equivalent, as follows from Proposition 2.2 in [26]: 
Proposition 2.1 A function $u$ solves Problem $P_{C}$ if and only if $(p, \mathbf{q})$ defined as

$$
(p, \mathbf{q})=\left(u,-\left(\nabla u+k(b(u)) \mathbf{e}_{z}\right)\right)
$$

solves Problem $P_{M}$. Moreover, in this case we have $p \in L^{2}\left(0, T ; H_{0}^{1}(\Omega)\right)$.

Proof In [26], $b(\cdot)$ is assumed Lipschitz continuous, which is more restrictive than (A2). However, the regularity of $u$ and $\mathbf{q}$ stated above allows us to prove the equivalence in the present setting as well. To do so, we simply have to follow the steps in [26]. The details are omitted here.

\section{The time discretization}

We now proceed with the time discretization for Problem $P_{M}$, which is achieved by the Euler implicit scheme. Let $N \geq 1$ be an integer giving the time step $\tau=T / N$. For a given $n \in\{1,2, \ldots, N\}$, with $t_{n}=n \tau$ we define the time discrete mixed variational problem:

Problem $P_{M}^{n}$. Let $p^{n-1}$ be given. Find $\left(p^{n}, \mathbf{q}^{n}\right) \in L^{2}(\Omega) \times H(\operatorname{div} ; \Omega)$ such that

$$
\begin{array}{r}
\left\langle b\left(p^{n}\right)-b\left(p^{n-1}\right), w\right\rangle+\tau\left\langle\nabla \cdot \mathbf{q}^{n}, w\right\rangle=0, \\
\left\langle\mathbf{q}^{n}, \mathbf{v}\right\rangle-\left\langle p^{n}, \nabla \cdot \mathbf{v}\right\rangle+\left\langle k\left(b\left(p^{n}\right)\right) \mathbf{e}_{z}, \mathbf{v}\right\rangle=0,
\end{array}
$$

for all $w \in L^{2}(\Omega)$, and $\mathbf{v} \in H(\operatorname{div} ; \Omega)$.

Initially we take $p^{0}=u_{I} \in L^{2}(\Omega)$.

Assuming $b(\cdot)$ Lipschitz continuous, the convergence of the time discrete numerical scheme is proven in [26] by showing the equivalence between the conformal and mixed forms of the temporal discretization. Then the proof is done for the conformal method. In this section we give a simplified convergence proof, which applies directly to the mixed time discretization given in (3.1), (3.2). Before doing so, it is worth noticing that the equivalence between the semidiscrete mixed and conformal schemes holds in the present generalized setting as well. To prove this, we simply have to proceed as in the proof of Proposition 2.3 in [26]. Furthermore, this equivalence also provides the existence of a solution for Problem $P_{M}^{n}$, as well as its uniqueness at least under a mild restriction on $\tau: \tau<4 / C_{k}$. This is due to the existence and uniqueness for the conformal problem that is equivalent to Problem $P_{M}^{n}$. In the case of a Lipschitz continuous nonlinearity $b(\cdot)$, these results are proven for example in [19], Chapter 4. If $b(\cdot)$ is only Hölder continuous, the existence can be obtained by approximating it in the $C^{0, \alpha}$ norm by a family of Lipschitz continuous functions $b_{\delta}(\cdot)$. The resulting regularized problems have unique solutions that are uniformly bounded in $H_{0}^{1}(\Omega)$. The weak limit $u$ of a sequence $\left\{u_{\delta_{n}}\right\}_{n \in \mathbb{N}}$ with $\delta_{n} \searrow 0$ solves Problem $P_{M}^{n}$, and uniqueness follows by standard energy arguments. We omit the details here and only mention that, as a result of this equivalence, $p^{n}$ has a better regularity:

$$
p^{n} \in H_{0}^{1}(\Omega) \text {. }
$$

In what follows we will make use of the elementary result below. 
Proposition 3.1 Let $\mathbf{a}_{k} \in \mathbb{R}^{d}(k \in\{1, \ldots, N\}, d \geq 1)$ be a set of d-dimensional vectors. We have

$$
2 \sum_{n=1}^{N}\left\langle\mathbf{a}_{n}, \sum_{k=1}^{n} \mathbf{a}_{k}\right\rangle=\left\|\sum_{n=1}^{N} \mathbf{a}_{n}\right\|^{2}+\sum_{n=1}^{N}\left\|\mathbf{a}_{n}\right\|^{2}
$$

Proof Since $\left\|\mathbf{a}_{k}\right\|^{2}=\left\langle\mathbf{a}_{k}, \mathbf{a}_{k}\right\rangle$, the proof follows by counting the terms in the left and right side.

To prove the convergence of the semidiscrete scheme (3.1), (3.2), we use the following stability estimates:

Lemma 3.2 Assuming (A1)-(A4), we have

$$
\begin{gathered}
\tau \sum_{n=1}^{N}\left\|p^{n}\right\|_{1}^{2}+\tau \sum_{n=1}^{N}\left\|\mathbf{q}^{n}\right\|^{2} \leq C, \\
\sum_{n=1}^{N}\left\langle b\left(p^{n}\right)-b\left(p^{n-1}\right), p^{n}-p^{n-1}\right\rangle+\tau \max _{n=1, \ldots, N}\left\|\mathbf{q}^{n}\right\|^{2} \\
+\tau \sum_{n=1}^{N}\left\|\mathbf{q}^{n}-\mathbf{q}^{n-1}\right\|^{2} \leq C \tau, \\
\tau \sum_{n=1}^{N}\left\|\nabla \cdot \mathbf{q}^{n}\right\|^{2} \leq C \tau^{-\frac{2(1-\alpha)}{1+\alpha} .}
\end{gathered}
$$

Proof We test (3.1) with $p^{n}$ and (3.2) with $\tau \mathbf{q}^{n}$, add the equalities and sum the resulting up for $n=1, \ldots, N$. This gives

$$
\sum_{n=1}^{N}\left\langle b\left(p^{n}\right)-b\left(p^{n-1}\right), p^{n}\right\rangle+\tau \sum_{n=1}^{N}\left\|\mathbf{q}^{n}\right\|^{2}+\tau \sum_{n=1}^{N}\left\langle k\left(b\left(p^{n}\right)\right) \mathbf{e}_{z}, \mathbf{q}^{n}\right\rangle=0
$$

The three terms in the above are denoted by $T_{1}, T_{2}$ and $T_{3}$. To estimate $T_{1}$ we notice that if $b(\cdot)$ satisfies the assumption (A2), for any reals $x$ and $y$ we have

$$
(b(x)-b(y)) x \geq \int_{y}^{x} s b^{\prime}(s) d s, \text { and } \int_{0}^{x} s b^{\prime}(s) d s \geq 0 .
$$


Together with the Hölder continuity of $b(\cdot)$ this gives

$$
\begin{aligned}
T_{1} & \geq \sum_{n=1}^{N} \int_{\Omega} \int_{p^{n-1}}^{p^{n}} s b^{\prime}(s) d s d x=\int_{\Omega} \int_{0}^{p^{N}} s b^{\prime}(s) d s d x-\int_{\Omega} \int_{0}^{p^{0}} s b^{\prime}(s) d s d x \\
& \geq-\int_{\Omega} \int_{0}^{p^{0}} s b^{\prime}(s) d s d x=\int_{\Omega} \int_{0}^{p^{0}} b(s)-b\left(p^{0}\right) d s d x \geq-\int_{\Omega} C_{b}\left|p^{0}\right|^{\alpha+1} d x .
\end{aligned}
$$

Since $p^{0}=u_{I} \in L^{2}(\Omega)$, for $\alpha=1$ we immediately obtain $T_{1} \geq-C$. The case $\alpha \in(0,1)$ is solved by applying Young's inequality

$$
|a b| \leq{\frac{|a|^{p}}{p}}^{p}+{\frac{|b|^{q}}{q}}^{q}
$$

with $p=\frac{2}{1+\alpha}$ and $q=\frac{2}{1-\alpha}$. We obtain again $T_{1} \geq-C$ for some constant $C>0$.

$T_{2}$ needs no further treatment, while for $T_{3}$ we apply the Cauchy inequality, as well as the inequality of means to prove the estimates for $\mathbf{q}$ in (3.5). For the $H^{1}$ estimates for $p$ we first notice that, by (3.3), (3.2) becomes:

$$
\left\langle\nabla p^{n}, \mathbf{v}\right\rangle=-\left\langle\mathbf{q}^{n}, \mathbf{v}\right\rangle-\left\langle k\left(b\left(p^{n}\right)\right) \mathbf{e}_{z}, \mathbf{v}\right\rangle,
$$

for all $\mathbf{v} \in H(\operatorname{div} ; \Omega)$. Since both $\mathbf{q}^{n}$ and $k\left(b\left(p^{n}\right)\right)$ are actually $L^{2}$, the above inequality holds for any $\mathbf{v} \in\left(L^{2}(\Omega)\right)^{d}$. This, together with the boundedness of $k(\cdot)$, the estimates for $\mathbf{q}^{n}$ and the inequality of Poincaré completes the proof of (3.5).

To prove (3.6), one simply has to follow the proof of Proposition 3.5 in [26]. Finally, for obtaining the estimate (3.7), we test (3.1) by $\nabla \cdot \mathbf{q}^{n}$. Applying the Cauchy inequality gives

$$
\tau\left\|\nabla \cdot \mathbf{q}^{n}\right\| \leq\left\|b\left(p^{n}\right)-b\left(p^{n-1}\right)\right\|
$$

yielding straightforwardly

$$
\tau \sum_{n=1}^{N}\left\|\nabla \cdot \mathbf{q}^{n}\right\|^{2} \leq \frac{1}{\tau} \sum_{n=1}^{N}\left\|b\left(p^{n}\right)-b\left(p^{n-1}\right)\right\|^{2} .
$$

If the Hölder exponent $\alpha$ in (A2) is 1 (thus if $b(\cdot)$ is Lipschitz continuous), the proof is concluded by the estimate in (3.6). For the case $\alpha \in(0,1)$ we first notice that (A2) and (3.6) immediately imply

$$
\sum_{j=1}^{n}\left\|b\left(p^{j}\right)-b\left(p^{j-1}\right)\right\|_{L^{1+1 / \alpha}(\Omega)}^{1+1 / \alpha} \leq C \tau .
$$


Further, with $r=\frac{2(1-\alpha)}{1+\alpha}$ we use the inequality (3.8) to estimate the sum on the right in (3.9) by

$$
\sum_{n=1}^{N}\left\|b\left(p^{n}\right)-b\left(p^{n-1}\right)\right\|^{2} \leq \frac{1}{\tau^{r}} \sum_{n=1}^{N}\left(\int_{\Omega} \frac{\tau}{p}^{r p} d x+\frac{1}{q}\left\|b\left(p^{n}\right)-b\left(p^{n-1}\right)\right\|_{L^{2 q}(\Omega)}^{2 q}\right) .
$$

With $p=\frac{\alpha+1}{1-\alpha}$ and $q=\frac{\alpha+1}{2 \alpha}$ and recalling (3.10) this gives

$$
\sum_{n=1}^{N}\left\|b\left(p^{n}\right)-b\left(p^{n-1}\right)\right\|^{2} \leq C \tau^{1-r}
$$

and the rest of the proof is straightforward.

In what follows we prove the convergence of the mixed time discrete scheme (3.1)(3.2). This extends the result stated in Theorem 4.6 of [26] to the case of Hölder continuous nonlinearities $b(\cdot)$. As follows from below, the present demonstration is simplified by giving up the convergence proof for the conformal scheme, as done in [26]. The error estimates are obtained now in a direct manner, which applies to mixed variational formulations with test functions in $L^{2}(\Omega)$.

For any time dependent function $f$ defined on the interval $[0, T]$, we first introduce the notations:

$$
\begin{aligned}
\bar{f}^{n} & =\frac{1}{\tau} \int_{t_{n-1}}^{t_{n}} f(t) d t, \\
f_{\Delta}(t) & =f^{n}, \quad \text { for } t \in\left(t_{n-1}, t_{n}\right],
\end{aligned}
$$

whenever $n \in\{1, \ldots, N\}$. For $n=0$ we take $\bar{f}^{0}=f(0)$. Recalling that $p \in$ $L^{2}\left(0, T ; H^{1}(\Omega)\right)$ by the equivalence between the Problems $P_{C}$ and $P_{M}$, and by (3.5), we have

$$
\sum_{n=1}^{N} \tau\left\|\bar{p}^{n}-p^{n}\right\|_{1}^{2} \leq \sum_{n=1}^{N} \int_{t_{n-1}}^{t_{n}}\left\|p(t)-p^{n}\right\|_{1}^{2} d t \leq C
$$

This further implies

$$
\sum_{n=1}^{N} \int_{t_{n-1}}^{t_{n}}\left\|\mathbf{q}(t)-\mathbf{q}^{n}\right\|^{2} d t \leq C
$$


yielding

$$
\sum_{n=1}^{N}\left\|\int_{t_{n-1}}^{t_{n}} \mathbf{q}(t)-\mathbf{q}^{n} d t\right\|^{2} \leq \tau \sum_{n=1}^{N} \int_{t_{n-1}}^{t_{n}}\left\|\mathbf{q}(t)-\mathbf{q}^{n}\right\|^{2} d t \leq C \tau
$$

Now we can proceed by estimating the error for the mixed time discrete scheme (3.1)-(3.2). In what follows we assume that $\tau$ is sufficiently small, so that the discrete Gronwall lemma can be applied.

Lemma 3.3 Assuming (A1)-(A4), for any $K=1, \ldots, N$ we have the estimates

$$
\sum_{n=1}^{K} \int_{t_{n-1}}^{t_{n}}\left\langle b(p(t))-b\left(p^{n}\right), p(t)-p^{n}\right\rangle d t+\left\|\sum_{n=1}^{K} \int_{t_{n-1}}^{t_{n}}\left(\mathbf{q}(t)-\mathbf{q}^{n}\right) d t\right\|^{2} \leq C \tau .
$$

Proof For any $n \in\{1, \ldots, N\},(3.1)$ immediately implies

$$
\left\langle b\left(p^{n}\right)-b\left(p^{0}\right), w\right\rangle+\tau\left\langle\nabla \cdot \sum_{k=1}^{n} \mathbf{q}^{k}, w\right\rangle=0,
$$

for all $w \in L^{2}(\Omega)$. Further, (2.8) and (2.9) can be rewritten as

$$
\begin{array}{r}
\left\langle b\left(p\left(t_{n}\right)\right)-b(p(0)), w\right\rangle+\left\langle\tau \sum_{k=1}^{n} \nabla \cdot \mathbf{q}^{k}, w\right\rangle=0, \\
\left\langle\overline{\mathbf{q}}^{n}, \mathbf{v}\right\rangle-\left\langle\bar{p}^{n}, \nabla \cdot \mathbf{v}\right\rangle+\left\langle\overline{k(b(p))}^{n} \mathbf{e}_{z}, \mathbf{v}\right\rangle=0,
\end{array}
$$

for all $w \in L^{2}(\Omega)$, respectively $\mathbf{v} \in H(\operatorname{div} ; \Omega)$. Subtracting now (3.14) from (3.15) and (3.2) from (3.16) and recalling that $p(0)=p^{0}$ gives

$$
\begin{array}{r}
\left\langle b\left(p\left(t_{n}\right)\right)-b\left(p^{n}\right), w\right\rangle+\left\langle\tau \sum_{k=1}^{n} \nabla \cdot\left\langle\overline{\mathbf{q}}^{k}-\mathbf{q}^{k}\right), w\right\rangle=0, \\
\left\langle\overline{\mathbf{q}}^{n}-\mathbf{q}^{n}, \mathbf{v}\right\rangle-\left\langle\bar{p}^{n}-p^{n}, \nabla \cdot \mathbf{v}\right\rangle+\left\langle\left(\overline{k(b(p))}^{n}-k\left(b\left(p^{n}\right)\right)\right) \mathbf{e}_{z}, \mathbf{v}\right\rangle=0,
\end{array}
$$

for all $w \in L^{2}(\Omega)$ and $\mathbf{v} \in H(\operatorname{div} ; \Omega)$. Taking now $w=\bar{p}^{n}-p^{n}$ in (3.17) and $\mathbf{v}=\tau \sum_{k=1}^{n}\left(\overline{\mathbf{q}}^{k}-\mathbf{q}^{k}\right) \in H(\operatorname{div} ; \Omega)$ in (3.18), and adding the resulting yields

$$
\begin{gathered}
\left\langle b\left(p\left(t_{n}\right)\right)-b\left(p^{n}\right), \bar{p}^{n}-p^{n}\right\rangle+\left\langle\overline{\mathbf{q}}^{n}-\mathbf{q}^{n}, \tau \sum_{k=1}^{n}\left(\overline{\mathbf{q}}^{k}-\mathbf{q}^{k}\right)\right\rangle \\
\left.+\left\langle\overline{(k(b(p))}^{n}-k\left(b\left(p^{n}\right)\right)\right) \mathbf{e}_{z}, \tau \sum_{k=1}^{n}\left(\overline{\mathbf{q}}^{k}-\mathbf{q}^{k}\right)\right\rangle=0 .
\end{gathered}
$$


Summing the above for $n=1, \ldots, K$ leads to

$$
\begin{aligned}
& \sum_{n=1}^{K}\left\langle b\left(p\left(t_{n}\right)\right)-b\left(p^{n}\right), \bar{p}^{n}-p^{n}\right\rangle+\tau \sum_{n=1}^{K}\left\langle\overline{\mathbf{q}}^{n}-\mathbf{q}^{n}, \sum_{k=1}^{n}\left(\overline{\mathbf{q}}^{k}-\mathbf{q}^{k}\right)\right\rangle \\
& \quad+\tau \sum_{n=1}^{K}\left\langle\left(\overline{k(b(p))}^{n}-k\left(b\left(p^{n}\right)\right)\right) \mathbf{e}_{z}, \sum_{k=1}^{n}\left(\overline{\mathbf{q}}^{k}-\mathbf{q}^{k}\right)\right\rangle=0 .
\end{aligned}
$$

We denote by $T_{1}, T_{2}$ and $T_{3}$ the terms in the above and estimate them separately. By (3.4), we have

$$
T_{2}=\frac{\tau}{2}\left\|\sum_{n=1}^{K}\left(\overline{\mathbf{q}}^{n}-\mathbf{q}^{n}\right)\right\|^{2}+\frac{\tau}{2} \sum_{n=1}^{K}\left\|\left(\overline{\mathbf{q}}^{n}-\mathbf{q}^{n}\right)\right\|^{2} .
$$

To estimate $T_{1}$, we first split it as

$$
\begin{aligned}
T_{1}= & \frac{1}{\tau} \sum_{n=1}^{K} \int_{t_{n-1}}^{t_{n}}\left\langle b\left(p\left(t_{n}\right)\right)-b(p(t)), p(t)-p^{n}\right\rangle d t \\
& +\frac{1}{\tau} \sum_{n=1}^{K} \int_{t_{n-1}}^{t_{n}}\left\langle b(p(t))-b\left(p^{n}\right), p(t)-p^{n}\right\rangle d t .
\end{aligned}
$$

Denoting the terms above by $T_{11}$ and $T_{12}$, since $b(\cdot)$ is nondecreasing we immediately get $T_{12}>0$. For $T_{11}$ we use (3.11) and the regularity of $\partial_{t} b(p)$ and $p$,

$$
\begin{aligned}
\left|T_{11}\right| & =\frac{1}{\tau}\left|\sum_{n=1}^{K} \int_{t_{n-1}}^{t_{n}}\left\langle\int_{t}^{t_{n}} \partial_{s} b(p)(s) d s, p(t)-p^{n}\right\rangle d t\right| \\
& \leq \frac{1}{\tau} \sum_{n=1}^{K} \int_{t_{n-1}}^{t_{n}} \int_{t}^{t_{n}}\left\|\partial_{s} b(p)\right\|_{-1}\left\|p(t)-p^{n}\right\|_{1} d s d t \\
& \leq \frac{1}{2 \tau} \sum_{n=1}^{K}\left(\int_{t_{n-1}}^{t_{n}}\left\|\partial_{s} b(p)\right\|_{-1} d s\right)^{2}+\frac{1}{2 \tau} \sum_{n=1}^{K}\left(\int_{t_{n-1}}^{t_{n}}\left\|p(t)-p^{n}\right\|_{1} d t\right)^{2} \\
& \leq \frac{1}{2}\left\{\int_{0}^{T}\left\|\partial_{t} b(p)\right\|_{-1}^{2} d t+\sum_{n=1}^{K} \int_{t_{n-1}}^{t_{n}}\left\|p(t)-p^{n}\right\|_{1}^{2} d t\right\} \leq C .
\end{aligned}
$$


Finally, the convection term $T_{3}$ gives

$$
\begin{aligned}
\left|T_{3}\right| & \leq \sum_{n=1}^{K}\left|\left\langle\left({\overline{k(b(p))^{n}}}^{n}-k\left(b\left(p^{n}\right)\right)\right) \mathbf{e}_{z}, \tau \sum_{k=1}^{n}\left(\overline{\mathbf{q}}^{k}-\mathbf{q}^{k}\right)\right\rangle\right| \\
& \leq \frac{1}{2 C_{k}} \sum_{n=1}^{K} \| \overline{k(b(p))}^{n}-k\left(b\left(p^{n}\right)\left\|^{2}+\frac{\tau^{2} C_{k}}{2} \sum_{n=1}^{K}\right\| \sum_{k=1}^{n}\left(\overline{\mathbf{q}}^{k}-\mathbf{q}^{k}\right) \|^{2}\right. \\
& =: T_{31}+T_{32} .
\end{aligned}
$$

For $T_{31}$ we use (A3) and obtain

$$
\begin{aligned}
T_{31} & =\frac{1}{2 \tau^{2} C_{k}} \sum_{n=1}^{K} \int_{\Omega}\left[\int_{t_{n-1}}^{t_{n}} k(b(p(t)))-k\left(b\left(p^{n}\right)\right) d t\right]^{2} d x \\
& \leq \frac{1}{2 \tau C_{k}} \sum_{n=1}^{K} \int_{\Omega} \int_{t_{n-1}}^{t_{n}}\left(k(b(p(t)))-k\left(b\left(p^{n}\right)\right)\right)^{2} d t d x \\
& \leq \frac{1}{2 \tau} \sum_{n=1}^{K} \int_{t_{n-1}}^{t_{n}}\left\langle b(p(t))-b\left(p^{n}\right), p(t)-p^{n}\right\rangle d t .
\end{aligned}
$$

Using (3.20)-(3.24) into (3.19) gives

$$
\begin{aligned}
& \frac{1}{2 \tau} \sum_{n=1}^{K} \int_{t_{n-1}}^{t_{n}}\left\langle b(p(t))-b\left(p^{n}\right), p(t)-p^{n}\right\rangle d t+\frac{\tau}{2}\left\|\sum_{n=1}^{K}\left(\overline{\mathbf{q}}^{n}-\mathbf{q}^{n}\right)\right\|^{2} \\
& \quad+\frac{\tau}{2} \sum_{n=1}^{K}\left\|\left(\overline{\mathbf{q}}^{n}-\mathbf{q}^{n}\right)\right\|^{2} \leq C+\frac{\tau^{2} C_{k}}{2} \sum_{n=1}^{K}\left\|\sum_{k=1}^{n}\left(\overline{\mathbf{q}}^{k}-\mathbf{q}^{k}\right)\right\|^{2},
\end{aligned}
$$

and the result follows by applying the discrete Gronwall lemma.

Remark 3.4 As follows from Gronwall's lemma, the constant $C$ appearing in the estimates proven above is depending exponentially on $T$. In the absence of convection there is no need for applying Gronwall's lemma, and the constant $C$ does not depend on $T$ anymore. In particular we obtain:

$$
\left\|\int_{0}^{t} \mathbf{q}(s)-\mathbf{q}_{\Delta} d s\right\|_{L^{\infty}\left(0, T ; L^{2}(\Omega)\right)}^{2} \leq C \tau .
$$


Remark 3.5 By (A2), the estimates in Lemma 3.3 immediately imply

$$
\int_{0}^{T}\left\|b(p(t))-b\left(p_{\Delta}(t)\right)\right\|_{L^{1+1 / \alpha}(\Omega)}^{1+1 / \alpha} d t \leq C \tau .
$$

The estimates in Lemma 3.3 can be improved under stronger assumptions on $b(u)$, and by ruling out the fast diffusion case. Specifically, we have the following:

\section{Corollary 3.6 Assuming}

$$
\partial_{t} b(u) \in L^{2}\left(0, T ; L^{2}(\Omega)\right) \quad \text { and } \quad b^{\prime}(\cdot) \geq C_{\text {inf }}>0,
$$

the estimates in Lemma 3.3 become optimal:

$$
\sum_{n=1}^{K} \int_{t_{n-1}}^{t_{n}}\left\langle b(p(t))-b\left(p^{n}\right), p(t)-p^{n}\right\rangle d t+\left\|\sum_{n=1}^{K} \int_{t_{n-1}}^{t_{n}}\left(\mathbf{q}(t)-\mathbf{q}^{n}\right) d t\right\|^{2} \leq C \tau^{2},
$$

for any $K=1, \ldots, N$.

Proof The proof follows the ideas in the demonstration of Lemma 3.3. The regularity of $\partial_{t} b(u)$ allows estimating $T_{11}$ as

$$
\begin{aligned}
\left|T_{11}\right| & =\frac{1}{\tau}\left|\sum_{n=1}^{K} \int_{t_{n-1}}^{t_{n}}\left\langle\int_{t}^{t_{n}} \partial_{s} b(p)(s) d s, p(t)-p^{n}\right\rangle d t\right| \\
& \leq \sum_{n=1}^{K} \int_{t_{n-1}}^{t_{n}} \int_{t}^{t_{n}} \frac{1}{2 \delta}\left\|\partial_{s} b(p(s))\right\|^{2}+\frac{\delta}{2 \tau^{2}}\left\|p(t)-p^{n}\right\|^{2} d s d t \\
& \leq \frac{\tau}{2 C_{i n f}}\left\|\partial_{S} b(p)\right\|_{L^{2}\left(0, T ; L^{2}(\Omega)\right)}^{2}+\sum_{n=1}^{K} \frac{C_{i n f}}{2 \tau} \int_{t_{n-1}}^{t_{n}}\left\|p(t)-p^{n}\right\|^{2} d t .
\end{aligned}
$$

By (3.27), the first term on the right in the above is bounded by $\tau C$ for some $C>0$. Further, for dealing with the remaining term we estimate from below the last term in (3.21) as follows

$$
\begin{aligned}
& \frac{1}{\tau} \sum_{n=1}^{K} \int_{t_{n-1}}^{t_{n}}\left\langle b(p(t))-b\left(p^{n}\right), p(t)-p^{n}\right\rangle d t \\
& \quad \geq \frac{1}{2 \tau} \sum_{n=1}^{K} \int_{t_{n-1}}^{t_{n}}\left\langle b(p(t))-b\left(p^{n}\right), p(t)-p^{n}\right\rangle d t+\frac{C_{i n f}}{2 \tau} \sum_{n=1}^{K} \int_{t_{n-1}}^{t_{n}}\left\|p(t)-p^{n}\right\|^{2} d t .
\end{aligned}
$$

Now the proof can be completed by the steps carried out in proving Lemma 3.3. 
Remark 3.7 Based on the equivalence with the conformal formulations and following [29], a similar result can be obtained in the absence of convection without assuming the $L^{2}$ regularity of $\partial_{t} b(u)$.

In the following we will make use of the following lemma:

Lemma 3.8 Given a $w \in L^{2}(\Omega)$, there exists $a \mathbf{v} \in H(\operatorname{div} ; \Omega)$ such that

$$
\nabla \cdot \mathbf{v}=w \text { and }\|\mathbf{v}\| \leq C\|w\|,
$$

with $C>0$ not depending on $w$.

Proof Let $u$ be the (weak) solution of the Poisson equation

$$
-\Delta u=w, \quad \text { in } \Omega,
$$

and having a vanishing trace on $\Gamma$. Testing the above equation by $u$ and recalling the Poincaré inequality, we immediately obtain $\|\nabla u\| \leq C\|w\|$. The result follows now by taking $\mathbf{v}=-\nabla u$.

The estimates in Lemma 3.3 can now be enriched.

Lemma 3.9 Assuming (A1)-(A4), for any $K=1, \ldots, N$ we have

$$
\begin{aligned}
\left\|\sum_{n=1}^{K} \int_{t_{n-1}}^{t_{n}} p(t)-p^{n} d t\right\|^{2} \leq & C\left\|\sum_{n=1}^{K} \int_{t_{n-1}}^{t_{n}} \mathbf{q}(t)-\mathbf{q}^{n} d t\right\|^{2} \\
& +C \sum_{n=1}^{K} \int_{t_{n-1}}^{t_{n}}\left\langle b(p(t))-b\left(p^{n}\right), p(t)-p^{n}\right\rangle d t .
\end{aligned}
$$

Proof Subtracting (3.2) from (3.16) and adding the result for $n=1, \ldots, K$, we end up with

$$
\left\langle\sum_{n=1}^{K}\left(\overline{\mathbf{q}}^{n}-\mathbf{q}^{n}\right), \mathbf{v}\right\rangle-\left\langle\sum_{n=1}^{K}\left(\bar{p}^{n}-p^{n}\right), \nabla \cdot \mathbf{v}\right\rangle+\left\langle\sum_{n=1}^{K}\left(\overline{k(b(p))}^{n}-k\left(b\left(p^{n}\right)\right)\right) \mathbf{e}_{z}, \mathbf{v}\right\rangle=0,
$$

for all $\mathbf{v} \in H(\operatorname{div} ; \Omega)$. Further, by Lemma 3.8, a $\mathbf{v} \in H(\operatorname{div} ; \Omega)$ exists such that (3.28) holds for $w=\sum_{n=1}^{K}\left(\bar{p}^{n}-p^{n}\right)$. Taking this $\mathbf{v}$ in the above yields

$$
\left\|\sum_{n=1}^{K}\left(\bar{p}^{n}-p^{n}\right)\right\|^{2}=\left\langle\sum_{n=1}^{K}\left(\overline{\mathbf{q}}^{n}-\mathbf{q}^{n}\right), \mathbf{v}\right\rangle+\left\langle\sum_{n=1}^{K}\left(\overline{k(b(p))}^{n}-k\left(b\left(p^{n}\right)\right)\right) \mathbf{e}_{z}, \mathbf{v}\right\rangle .
$$


Since $\|\mathbf{v}\| \leq C\|w\|$, it follows that

$$
\left\|\sum_{n=1}^{K}\left(\bar{p}^{n}-p^{n}\right)\right\|^{2} \leq 2 C\left\{\left\|\sum_{n=1}^{K}\left(\overline{\mathbf{q}}^{n}-\mathbf{q}^{n}\right)\right\|^{2}+\left\|\sum_{n=1}^{K}\left(\overline{k(b(p))}^{n}-k\left(b\left(p^{n}\right)\right)\right) \mathbf{e}_{z}\right\|^{2}\right\}
$$

The last term in the above is estimated by

$$
\begin{aligned}
T & \leq \frac{1}{\tau^{2}} \int_{\Omega}\left(\sum_{n=1}^{K} \int_{t_{n-1}}^{t_{n}} k(b(p(t)))-k\left(b\left(p^{n}\right)\right) d t\right)^{2} d x \\
& \leq \frac{K}{\tau} \int_{\Omega} \sum_{n=1}^{K} \int_{t_{n-1}}^{t_{n}}\left(k(b(p(t)))-k\left(b\left(p^{n}\right)\right)\right)^{2} d t d x \\
& \stackrel{(A 3)}{\leq} \frac{K C_{k}}{\tau} \sum_{n=1}^{K} \int_{t_{n-1}}^{t_{n}}\left\langle b(p(t))-b\left(p^{n}\right), p(t)-p^{n}\right\rangle d t .
\end{aligned}
$$

In this way we obtain

$$
\begin{aligned}
\left\|\sum_{n=1}^{K}\left(\bar{p}^{n}-p^{n}\right)\right\|^{2} \leq & C \frac{1}{\tau^{2}} \sum_{n=1}^{K} \int_{t_{n-1}}^{t_{n}}\left\langle b(p(t))-b\left(p^{n}\right), p(t)-p^{n}\right\rangle d t \\
& +C\left\|\sum_{n=1}^{K}\left(\overline{\mathbf{q}}^{n}-\mathbf{q}^{n}\right)\right\|^{2} .
\end{aligned}
$$

The result follows straightforwardly, multiplying the above by $\tau^{2}$.

Summarizing the estimates in the Lemmas 3.3 and 3.9, we obtain the following theorem:

Theorem 3.10 Assuming (A1)-(A4), with $(p, \mathbf{q})$ and $\left(p^{n}, \mathbf{q}^{n}\right)$ solving Problem $P_{M}$, respectively Problem $P_{M}^{n}$ with $n=1, \ldots, N$, for any $K=1, \ldots, N$ we have

$$
\begin{aligned}
& \sum_{n=1}^{K} \int_{t_{n-1}}^{t_{n}}\left\langle b(p(t))-b\left(p^{n}\right), p(t)-p^{n}\right\rangle d t \\
& \quad+\left\|\sum_{n=1}^{K} \int_{t_{n-1}}^{t_{n}}\left(p(t)-p^{n}\right) d t\right\|^{2}+\left\|\sum_{n=1}^{K} \int_{t_{n-1}}^{t_{n}}\left(\mathbf{q}(t)-\mathbf{q}^{n}\right) d t\right\|^{2} \leq C \tau .
\end{aligned}
$$




\section{The fully discrete mixed discretization}

In this section we proceed by estimating the error for the fully discrete approximation. This is done first for the flux variable $\mathbf{q}$, and then for the $p$ unknown. In doing so we let $\mathcal{T}_{h}$ be a regular decomposition of $\Omega \subset \mathbb{R}^{d}$ into closed $d$-simplices; $h$ stands for the mesh-size (see [12]). Here we assume $\bar{\Omega}=\cup_{T \in \mathcal{T}_{h}} T$, hence $\Omega$ is polygonal. Thus we neglect the errors caused by an approximation of a nonpolygonal domain and avoid an excess of technicalities (a complete analysis in this sense can be found in [21]).

The discrete subspaces $W_{h} \times V_{h} \subset L^{2}(\Omega) \times H($ div; $\Omega)$ are defined as

$$
\begin{aligned}
W_{h} & :=\left\{p \in L^{2}(\Omega) \mid p \text { is constant on each element } T \in \mathcal{T}_{h}\right\}, \\
V_{h} & :=\left\{\mathbf{q} \in H(\operatorname{div} ; \Omega)|\mathbf{q}| T=\mathbf{a}+b \mathbf{x} \text { for all } T \in \mathcal{T}_{h}\right\}
\end{aligned}
$$

So $W_{h}$ denotes the space of piecewise constant functions, while $V_{h}$ is the $R T_{0}$ space (see [11]). Notice that $\nabla \cdot \mathbf{q} \in W_{h}$ for any $\mathbf{q} \in V_{h}$.

In what follows we make use of the usual $L^{2}$ projector:

$$
P_{h}: L^{2}(\Omega) \rightarrow W_{h}, \quad\left\langle P_{h} w-w, w_{h}\right\rangle=0,
$$

for all $w_{h} \in W_{h}$. Furthermore, a projector $\Pi_{h}$ can be defined on $\left(H^{1}(\Omega)\right)^{d}$ (see $[11$, p. 131]) such that

$$
\Pi_{h}:\left(H^{1}(\Omega)\right)^{d} \rightarrow V_{h}, \quad\left\langle\nabla \cdot\left(\Pi_{h} \mathbf{v}-\mathbf{v}\right), w_{h}\right\rangle=0,
$$

for all $w_{h} \in W_{h}$. Following [25], p. 237, this operator can be extended to $H$ (div; $\Omega$ ). For the above operators there holds

$$
\begin{gathered}
\left\|w-P_{h} w\right\| \leq C h\|w\|_{1}, \\
\left\|\mathbf{v}-\Pi_{h} \mathbf{v}\right\| \leq C h\|\mathbf{v}\|_{1}
\end{gathered}
$$

for any $w \in H^{1}(\Omega)$ and $\mathbf{v} \in\left(H^{1}(\Omega)\right)^{d}$.

The following technical lemma is proven in [33] (see also [31, p. 38]).

Lemma 4.1 Assuming (Al) and given a $f_{h} \in W_{h}, a \mathbf{v}_{h} \in V_{h}$ exists such that

$$
\nabla \cdot \mathbf{v}_{h}=f_{h} \text { and }\left\|\mathbf{v}_{\mathrm{h}}\right\| \leq \mathrm{C}\left\|\nabla \cdot \mathbf{v}_{\mathrm{h}}\right\|
$$

with $C>0$ being a constant not depending on $h, f_{h}$, or $\mathbf{v}_{h}$.

Further we will make use also of the following stability estimates. 
Lemma 4.2 Assume (A1)-(A4), and let $p^{n}$ be the first component in the solution pair of Problem $P_{M}^{n}$. With $n=1, \ldots, N$ there holds

$$
\begin{aligned}
\tau \sum_{n=1}^{N}\left\|p^{n}-P_{h} p^{n}\right\|_{L^{1+\alpha}(\Omega)}^{1+\alpha} \leq C h^{1+\alpha} & \\
\tau \sum_{n=1}^{N}\left\|p^{n}-P_{h} p^{n}\right\|_{L^{1+\alpha}(\Omega)}^{1+\alpha} \leq C & \left.\leq \tau^{2}+h^{2} \tau^{-\frac{2(1-\alpha)}{1+\alpha}}\right) .
\end{aligned}
$$

Proof For $\alpha=1$ the proof is straightforward, by using the estimates in (4.4) and (3.5). For $\alpha \in(0,1)$, using the imbedding Theorem 2.8 in [2], p. 25, Young's inequality and (4.4) we obtain

$$
\begin{aligned}
\tau \sum_{n=1}^{N}\left\|p^{n}-P_{h} p^{n}\right\|_{L^{1+\alpha}(\Omega)}^{1+\alpha} & \leq C \tau h^{1+\alpha} \sum_{n=1}^{N}\left\|p^{n}\right\|_{1}^{1+\alpha} \\
& \leq C h^{1+\alpha} \sum_{n=1}^{N}\left(\frac{\tau^{(1-r) s}}{s}+\frac{\tau^{r l}}{l}\left\|p^{n}\right\|_{1}^{(1+\alpha) l}\right)
\end{aligned}
$$

With $l=\frac{2}{1+\alpha}, s=\frac{2}{1-\alpha}$ and $r=\frac{1}{l}$, the first estimate follows by (3.5).

The second estimate follows from the first one by applying Young's inequality. We omit the details here.

With $n=1, \ldots, N$, the fully discrete problems can be defined:

Problem $P_{M}^{n, h}$. Let $p_{h}^{n-1}$ be given. Find $\left(p_{h}^{n}, \mathbf{q}_{h}^{n}\right) \in W_{h} \times V_{h}$ such that

$$
\begin{aligned}
\left\langle b\left(p_{h}^{n}\right)-b\left(p_{h}^{n-1}\right), w_{h}\right\rangle+\tau\left\langle\nabla \cdot \mathbf{q}_{h}^{n}, w_{h}\right\rangle & =0 \\
\left\langle\mathbf{q}_{h}^{n}, \mathbf{v}_{h}\right\rangle-\left\langle p_{h}^{n}, \nabla \cdot \mathbf{v}_{h}\right\rangle+\left\langle k\left(b\left(p_{h}^{n}\right)\right) \mathbf{e}_{z}, \mathbf{v}_{h}\right\rangle & =0
\end{aligned}
$$

for all $w_{h} \in W_{h}$ and $\mathbf{v}_{h} \in V_{h}$.

Initially we take a $p_{h}^{0} \in W_{h}$ such that it satisfies the condition $b\left(p_{h}^{0}\right)=P_{h} b\left(u_{I}\right)$ on any $T \in \mathcal{T}_{h}$. With this choice we obtain for all $w_{h} \in W_{h}$

$$
\left\langle b\left(p_{h}^{0}\right), w_{h}\right\rangle=\left\langle b\left(u_{I}\right), w_{h}\right\rangle=\left\langle b\left(p^{0}\right), w_{h}\right\rangle .
$$

Lemma 4.3 Assuming (A1)-(A4), with $\left(p^{n}, \mathbf{q}^{n}\right)$ and $\left(p_{h}^{n}, \mathbf{q}_{h}^{n}\right)$ solving Problem $P_{M}^{n}$, respectively Problem $P_{M}^{n, h}$ with $n=1, \ldots, N$, for any $K=1, \ldots, N$ we have

$$
\begin{aligned}
& \sum_{n=1}^{K}\left\{\left\langle b\left(p^{n}\right)-b\left(p_{h}^{n}\right), p^{n}-p_{h}^{n}\right\rangle+\left\|b\left(p^{n}\right)-b\left(p_{h}^{n}\right)\right\|_{L^{1+1 / \alpha}(\Omega)}^{1+1 / \alpha}\right\} \\
& \quad+\tau\left\|\sum_{n=1}^{K}\left(\Pi_{h} \mathbf{q}^{n}-\mathbf{q}_{h}^{n}\right)\right\|^{2} \leq C \sum_{n=1}^{K}\left\{\left\|\mathbf{q}^{n}-\Pi_{h} \mathbf{q}^{n}\right\|^{2}+\left\|P_{h} p^{n}-p^{n}\right\|_{L^{1+\alpha}(\Omega)}^{1+\alpha}\right\} .
\end{aligned}
$$


Proof From (4.7) we immediately obtain

$$
\left\langle b\left(p_{h}^{n}\right)-b\left(p_{h}^{0}\right), w_{h}\right\rangle+\tau \sum_{j=1}^{n}\left\langle\nabla \cdot \mathbf{q}_{h}^{j}, w_{h}\right\rangle=0
$$

for all $w_{h} \in W_{h}$ and for any $n=1, \ldots, N$. Subtracting (4.11) from (3.14), respectively (4.8) from (3.2), and recalling the definition of the projectors in (4.2) and (4.3), and using also (4.9) we end up with

$$
\begin{aligned}
& \left\langle b\left(p^{n}\right)-b\left(p_{h}^{n}\right), w_{h}\right\rangle+\tau \sum_{j=1}^{n}\left\langle\nabla \cdot \Pi_{h}\left(\mathbf{q}^{j}-\mathbf{q}_{h}^{j}\right), w_{h}\right\rangle=0 \\
& \left\langle\mathbf{q}^{n}-\mathbf{q}_{h}^{n}, \mathbf{v}_{h}\right\rangle-\left\langle P_{h} p^{n}-p_{h}^{n}, \nabla \cdot \mathbf{v}_{h}\right\rangle+\left\langle\left( k\left(b\left(p^{n}\right)\right)\right.\right. \\
& \left.\left.\quad-k\left(b\left(p_{h}^{n}\right)\right)\right) \mathbf{e}_{z}, \mathbf{v}_{h}\right\rangle=0
\end{aligned}
$$

for all $w_{h} \in W_{h}$ and $\mathbf{v}_{h} \in V_{h}$. Taking now $w_{h}=P_{h} p^{n}-p_{h}^{n} \in W_{h}$, and $\mathbf{v}_{h}=$ $\tau \sum_{j=1}^{n}\left(\Pi_{h} \mathbf{q}^{j}-\mathbf{q}_{h}^{j}\right) \in V_{h}$ into (4.12), respectively (4.13), adding the resulting and summing up for $n=1, \ldots, K$ with $K \leq N$ gives

$$
\begin{aligned}
& \sum_{n=1}^{K}\left\langle b\left(p^{n}\right)-b\left(p_{h}^{n}\right), P_{h} p^{n}-p_{h}^{n}\right\rangle+\tau \sum_{n=1}^{K}\left\langle\mathbf{q}^{n}-\mathbf{q}_{h}^{n}, \sum_{j=1}^{n}\left(\Pi_{h} \mathbf{q}^{j}-\mathbf{q}_{h}^{j}\right)\right\rangle \\
& \quad+\tau \sum_{n=1}^{K}\left\langle\left(k\left(b\left(p^{n}\right)\right)-k\left(b\left(p_{h}^{n}\right)\right)\right) \mathbf{e}_{z}, \sum_{j=1}^{n} \Pi_{h} \mathbf{q}^{j}-\mathbf{q}_{h}^{j}\right\rangle=0 .
\end{aligned}
$$

Now we proceed by estimating separately the terms in the above, denoted $T_{1}, T_{2}$ and $T_{3}$. It is worth mentioning that the estimates for $T_{2}$ and $T_{3}$ are obtained as in Proposition 4.10, p. 1470 in [26]. However, since $b(\cdot)$ is only Hölder continuous here, and not necessarily Lipschitz, $T_{1}$ requires a special attention. We start by writing

$$
\begin{aligned}
T_{1} & =\sum_{n=1}^{K}\left\langle b\left(p^{n}\right)-b\left(p_{h}^{n}\right), p^{n}-p_{h}^{n}\right\rangle+\sum_{n=1}^{K}\left\langle b\left(p^{n}\right)-b\left(p_{h}^{n}\right), P_{h} p^{n}-p^{n}\right\rangle \\
& =: T_{11}+T_{12} .
\end{aligned}
$$

The first term above is positive. Moreover, by (A2) we have

$$
\begin{aligned}
T_{11} \geq & \frac{1}{2} \sum_{n=1}^{K}\left\langle b\left(p^{n}\right)-b\left(p_{h}^{n}\right), p^{n}-p_{h}^{n}\right\rangle \\
& +\frac{C_{b}^{-\frac{1}{\alpha}}}{2} \sum_{n=1}^{K}\left\|b\left(p^{n}\right)-b\left(p_{h}^{n}\right)\right\|_{L^{1+\frac{1}{\alpha}}(\Omega)}^{1+\frac{1}{\alpha}} .
\end{aligned}
$$


For $T_{12}$ we use Young's inequality and obtain with $\delta=\left(4^{\alpha} C_{b}\right)^{-\frac{1}{1+\alpha}}\left(1+\frac{1}{\alpha}\right)^{\frac{\alpha}{1+\alpha}}>0$ :

$$
\begin{aligned}
T_{12} \leq & \frac{\delta^{1+\frac{1}{\alpha}}}{1+\frac{1}{\alpha}} \sum_{n=1}^{K}\left\|b\left(p^{n}\right)-b\left(p_{h}^{n}\right)\right\|_{L^{1+\frac{1}{\alpha}}(\Omega)}^{1+\frac{1}{\alpha}} \\
& +\frac{\delta^{-(1+\alpha)}}{1+\alpha} \sum_{n=1}^{K}\left\|P_{h} p^{n}-p^{n}\right\|_{L^{1+\alpha}(\Omega)}^{1+\alpha}
\end{aligned}
$$

As in Proposition 4.10, p. 1470 in [26], we use (3.4) and rewrite $T_{2}$ as

$$
\begin{aligned}
T_{2}= & \sum_{n=1}^{K}\left\langle\mathbf{q}^{n}-\Pi_{h} \mathbf{q}^{n}, \tau \sum_{j=1}^{n} \Pi_{h} \mathbf{q}^{j}-\mathbf{q}_{h}^{j}\right\rangle \\
& +\frac{\tau}{2}\left\|\sum_{n=1}^{K}\left(\Pi_{h} \mathbf{q}^{n}-\mathbf{q}_{h}^{n}\right)\right\|^{2}+\frac{\tau}{2} \sum_{n=1}^{K}\left\|\Pi_{h} \mathbf{q}^{n}-\mathbf{q}_{h}^{n}\right\|^{2} .
\end{aligned}
$$

The first term on the right, denoted $T_{21}$, is estimated by

$$
\left|T_{21}\right| \leq \frac{1}{2} \sum_{n=1}^{K}\left\|\mathbf{q}^{n}-\Pi_{h} \mathbf{q}^{n}\right\|^{2}+\frac{\tau^{2}}{2} \sum_{n=1}^{K}\left\|\sum_{j=1}^{n}\left(\Pi_{h} \mathbf{q}^{j}-\mathbf{q}_{h}^{j}\right)\right\|^{2} .
$$

Using (A3), $T_{3}$ gives for any $\delta>0$

$$
\left|T_{3}\right| \leq \frac{C_{k} \delta}{2} \sum_{n=1}^{K}\left\langle b\left(p^{n}\right)-b\left(p_{h}^{n}\right), p^{n}-p_{h}^{n}\right\rangle+\frac{\tau^{2}}{2 \delta} \sum_{n=1}^{K}\left\|\sum_{j=1}^{n}\left(\Pi_{h} \mathbf{q}^{j}-\mathbf{q}_{h}^{j}\right)\right\|^{2} .
$$

Inserting (4.15)-(4.20) into (4.14) and choosing $\delta$ properly leads to

$$
\begin{aligned}
& \sum_{n=1}^{K}\left\langle b\left(p^{n}\right)-b\left(p_{h}^{n}\right), p^{n}-p_{h}^{n}\right\rangle \\
& \quad+\tau\left\|\sum_{n=1}^{K}\left(\Pi_{h} \mathbf{q}^{n}-\mathbf{q}_{h}^{n}\right)\right\|^{2}+\sum_{n=1}^{K}\left\|b\left(p^{n}\right)-b\left(p_{h}^{n}\right)\right\|_{L^{1+\frac{1}{\alpha}}(\Omega)}^{1+\frac{1}{\alpha}} \\
& \quad \leq C \sum_{n=1}^{K}\left\{\left\|\mathbf{q}^{n}-\Pi_{h} \mathbf{q}^{n}\right\|^{2}+\left\|P_{h} p^{n}-p^{n}\right\|_{L^{1+\alpha}(\Omega)}^{1+\alpha}+\tau^{2}\left\|\sum_{j=1}^{n}\left(\Pi_{h} \mathbf{q}^{j}-\mathbf{q}_{h}^{j}\right)\right\|^{2}\right\} .
\end{aligned}
$$

Finally, (4.10) follows applying the discrete Gronwall lemma.

The above estimates for the flux error can be completed by estimates in $p$. To this aim we can proceed as in Proposition 4.12, p. 1472 in [26]. The main idea of the proof is to use Lemma 4.1. We omit the details here. 
Lemma 4.4 Under the assumptions of Lemma 4.3, for any $K=1, \ldots, N$ we have

$$
\begin{aligned}
& \tau\left\|\sum_{n=1}^{K}\left(P_{h} p^{n}-p_{h}^{n}\right)\right\|^{2} \leq C\left\{\sum_{n=1}^{K}\left\langle b\left(p^{n}\right)-b\left(p_{h}^{n}\right), p^{n}-p_{h}^{n}\right\rangle\right. \\
& \left.+\tau\left\|\sum_{n=1}^{K}\left(\Pi_{h} \mathbf{q}^{n}-\mathbf{q}_{h}^{n}\right)\right\|^{2}+\sum_{n=1}^{K}\left\|\mathbf{q}^{n}-\Pi_{h} \mathbf{q}^{n}\right\|^{2}\right\} .
\end{aligned}
$$

The error estimates between the time discrete and the fully discrete solution provided in Lemma 4.3 and Lemma 4.4 can be comprised in the following theorem.

Theorem 4.5 Assuming (A1)-(A4), let $\left(p^{n}, \mathbf{q}^{n}\right) \in L^{2}(\Omega) \times H(\operatorname{div} ; \Omega)$ and $\left(p_{h}^{n}, \mathbf{q}_{h}^{n}\right) \in$ $W_{h} \times V_{h}$ solve the problems $P_{M}^{n}$, respectively $P_{M}^{n, h}$, where $n=1, \ldots, N$. For any $K=1, \ldots, N$ we have

$$
\begin{aligned}
& \sum_{n=1}^{K}\left\{\left\langle b\left(p^{n}\right)-b\left(p_{h}^{n}\right), p^{n}-p_{h}^{n}\right\rangle+\left\|b\left(p^{n}\right)-b\left(p_{h}^{n}\right)\right\|_{L^{1+\frac{1}{\alpha}}(\Omega)}^{1+\frac{1}{\alpha}}\right\} \\
& \quad+\tau\left\|\sum_{n=1}^{K}\left(P_{h} p^{n}-p_{h}^{n}\right)\right\|^{2}+\tau\left\|\sum_{n=1}^{K}\left(\Pi_{h} \mathbf{q}^{n}-\mathbf{q}_{h}^{n}\right)\right\|^{2} \\
& \quad \leq C \sum_{n=1}^{K}\left\{\left\|\mathbf{q}^{n}-\Pi_{h} \mathbf{q}^{n}\right\|^{2}+\left\|P_{h} p^{n}-p^{n}\right\|_{L^{1+\alpha}(\Omega)}^{1+\alpha}\right\} .
\end{aligned}
$$

The last term in the above is bounded, as follows from Lemma 4.2. For the first term on the right we make the following assumption

(A5) $\mathbf{q}^{n} \in H^{1}(\Omega)^{d}$ for all $n=1, \ldots, N$ and $\sum_{n=1}^{N} \tau\left\|\mathbf{q}^{n}\right\|_{1}^{2} \leq C \tau^{-\frac{2(1-\alpha)}{1+\alpha}}$.

Remark 4.6 Notice that the above estimate involves a negative exponent of the time step $\tau$. It is suggested by the estimate (3.7), obtained for $\nabla \cdot \mathbf{q}^{n}$. Here we assume a similar bound for all partial derivatives of $\mathbf{q}^{n}$.

Remark 4.7 Assumption (A5) is automatically fulfilled in the case of one spatial dimension, when the spaces $H(\operatorname{div} ; \Omega)$ and $H^{1}(\Omega)$ coincide. In the multi dimensional case and in the absence of convection, one can follow the ideas in proving Theorem 1, p. 309 and Theorem 4, p. 317 in [15] to show that

$$
\left\|p^{n}\right\|_{2}^{2} \leq \frac{1}{\tau^{2}}\left\|\left(b\left(p^{n}\right)-b\left(p^{n-1}\right)\right)\right\|^{2}
$$

Then the equivalence with the mixed formulation gives

$$
\left\|\mathbf{q}^{n}\right\|_{1}^{2} \leq \frac{1}{\tau^{2}}\left\|\left(b\left(u^{n}\right)-b\left(u^{n-1}\right)\right)\right\|^{2} .
$$


This is similar to (3.9), with $H^{1}$ replacing $H(\operatorname{div} ; \Omega)$. Next, proceeding exactly as in Lemma 3.2 one can show (A5). The same proof can be extended to the case with convection by assuming $\left|(k(b))^{\prime}\right| \leq C$. Similarly, according to [19], Chapter 4 , if $\Omega$ has smooth boundaries, whereas $k(\cdot)$ is differentiable and $b(\cdot)$ is Lipschitz, then we also have $\mathbf{q}^{n} \in H^{1}$.

Using now Theorems 3.10 and 4.5, the projection estimates (4.4), as well as the inequality (3.26) and the stability estimates we end up with the error estimates for the fully discrete mixed finite element scheme:

Theorem 4.8 Assuming (A1)-(A5), for any $K=1, \ldots, N$ we have

$$
\begin{aligned}
& \left.\sum_{n=1}^{K} \int_{t_{n-1}}^{t_{n}} \| b(p(t))-b\left(p_{h}^{n}\right)\right)\left\|_{L^{1+\frac{1}{\alpha}}(\Omega)}^{1+\frac{1}{\alpha}} d t+\right\| \sum_{n=1}^{K} \int_{t_{n-1}}^{t_{n}}\left(p(t)-p_{h}^{n}\right) d t \|^{2} \\
& +\left\|\sum_{n=1}^{K} \int_{t_{n-1}}^{t_{n}}\left(\mathbf{q}(t)-\mathbf{q}_{h}^{n}\right) d t\right\|^{2} \leq C\left(\tau+h^{2} \tau^{-\frac{2(1-\alpha)}{1+\alpha}}\right) .
\end{aligned}
$$

Remark 4.9 As in Corollary 3.6, allowing only the slow diffusion case and assuming (A5), we obtain

$$
\begin{aligned}
& \left.\sum_{n=1}^{K} \int_{t_{n-1}}^{t_{n}} \| b(p(t))-b\left(p_{h}^{n}\right)\right)\left\|_{L^{1+\frac{1}{\alpha}}(\Omega)}^{1+\frac{1}{\alpha}} d t+\right\| \sum_{n=1}^{K} \int_{t_{n-1}}^{t_{n}}\left(p(t)-p_{h}^{n}\right) d t \|^{2} \\
& +\left\|\sum_{n=1}^{K} \int_{t_{n-1}}^{t_{n}}\left(\mathbf{q}(t)-\mathbf{q}_{h}^{n}\right) d t\right\|^{2} \leq C\left(\tau^{2}+h^{2} \tau^{-\frac{2(1-\alpha)}{1+\alpha}}\right) .
\end{aligned}
$$

For $\tau=h^{\frac{1+\alpha}{2}}$ this gives a convergence of order $h^{1+\alpha}$.

\section{Numerical results}

We test the considered numerical scheme on the following equation:

$$
\partial_{t} u^{1 / m}-\Delta u=0, \quad \text { in } \Omega \times(0, T],
$$

where $m>1$ is a given parameter, $\Omega=[0, L] \times[0, L]$ and $T>0$ the final time. All the computations have been performed in the software package $U G$ (see [8]). In this setting, assumption (A2) is fulfilled with $\alpha=1 / \mathrm{m}$. The above equation is derived in a straightforward manner from the porous medium equation, a typical slow diffusion model. With appropriate initial and boundary conditions, the equation above admits 
Table 1 Numerical results (final time $\mathrm{T}=2, m=2$ )

\begin{tabular}{llllll}
\hline $\mathrm{N}$ & $\tau$ & $\mathrm{h}$ & Error & $\tau^{2}+h^{1.5}$ & Convergence order \\
\hline 1 & 0.333 & 0.2 & $1.173610 \mathrm{e}-04$ & 0.20033172 & - \\
2 & 0.181818 & 0.1 & $3.739292 \mathrm{e}-05$ & 0.06468056 & 3.1386 \\
3 & 0.10526316 & 0.05 & $1.305061 \mathrm{e}-05$ & 0.02226067 & 2.8652 \\
4 & 0.0625 & 0.025 & $4.713953 \mathrm{e}-06$ & 0.00785910 & 2.7685 \\
5 & 0.037 & 0.0125 & $1.675419 \mathrm{e}-06$ & 0.00276654 & 2.8136 \\
\hline
\end{tabular}

Table 2 Numerical results (final time $\mathrm{T}=200, m=2$ )

\begin{tabular}{llllll}
\hline $\mathrm{N}$ & $\tau$ & $\mathrm{h}$ & Error & $\tau^{2}+h^{1.5}$ & Convergence order \\
\hline 1 & 0.333 & 0.2 & $1.915036 \mathrm{e}-04$ & 0.20033172 & - \\
2 & 0.181818 & 0.1 & $5.991603 \mathrm{e}-05$ & 0.06468056 & 3.1962 \\
3 & 0.10526316 & 0.05 & $2.074323 \mathrm{e}-05$ & 0.02226067 & 2.8885 \\
4 & 0.0625 & 0.025 & $7.469926 \mathrm{e}-06$ & 0.00785910 & 2.7769 \\
5 & 0.037 & 0.0125 & $2.660661 \mathrm{e}-06$ & 0.00276654 & 2.8075 \\
\hline
\end{tabular}

Table 3 Numerical results (final time $\mathrm{T}=2, m=4$ )

\begin{tabular}{llllll}
\hline $\mathrm{N}$ & $\tau$ & $\mathrm{h}$ & Error & $\tau^{2}+h^{1.25}$ & Convergence order \\
\hline 1 & 0.4 & 0.2 & $4.075752 \mathrm{e}-05$ & 0.29374806 & - \\
2 & 0.25 & 0.1 & $1.684891 \mathrm{e}-05$ & 0.11873413 & 2.4190 \\
3 & 0.1538 & 0.05 & $6.672659 \mathrm{e}-06$ & 0.04729798 & 2.5251 \\
4 & 0.1 & 0.025 & $2.899402 \mathrm{e}-06$ & 0.01994088 & 2.3014 \\
5 & 0.0645 & 0.0125 & $1.228340 \mathrm{e}-06$ & 0.00833988 & 2.3604 \\
\hline
\end{tabular}

the similarity solution (see [6]):

$$
u(t, x, y)=\frac{1}{t+1}\left[1-\frac{m-1}{4 m^{2}} \frac{x^{2}+y^{2}}{(t+1)^{1 / m}}\right]_{+}^{1 /(m-1)}
$$

The computations are performed for $m=2, m=4, L=1, T=2$ and $T=200$, and the errors are given in the Tables $1-4$. The initial grid is uniform, with $h=0.2$. This grid is then refined successively by halving $h$. Correspondingly, the time step is taken $\tau=h^{(m+1) / 2 m}$, and we compute the errors as given in (4.23). As revealed in the Tables $1-4$, the numerical results are confirming the theoretically estimated convergence order of $\tau^{2}+h^{(m+1) / m}$, since the reduction factor is close to $2^{(m+1) / m}$ at each refinement.

In the second numerical example we add a source term to the Eq. (5.1):

$$
\partial_{t} b(u)-\Delta u=f \quad \text { in } \Omega \times(0, T] .
$$


Table 4 Numerical results (final time $\mathrm{T}=200, m=4$ )

\begin{tabular}{llllll}
\hline $\mathrm{N}$ & $\tau$ & $\mathrm{h}$ & Error & $\tau^{2}+h^{1.25}$ & Convergence order \\
\hline 1 & 0.4 & 0.2 & $7.741041 \mathrm{e}-05$ & 0.29374806 & - \\
2 & 0.25 & 0.1 & $3.131379 \mathrm{e}-05$ & 0.11873413 & 2.4721 \\
3 & 0.1538 & 0.05 & $1.225694 \mathrm{e}-05$ & 0.04729798 & 2.5548 \\
4 & 0.1 & 0.025 & $5.298637 \mathrm{e}-06$ & 0.01994088 & 2.3132 \\
5 & 0.0645 & 0.0125 & $2.244762 \mathrm{e}-06$ & 0.00833988 & 2.3604 \\
\hline
\end{tabular}

Table 5 Numerical results (final time $\mathrm{T}=1, m=2$ )

\begin{tabular}{lllll}
\hline $\mathrm{N}$ & $\tau$ & $\mathrm{h}$ & Error & Reduction \\
\hline 1 & 0.1 & 0.1 & $2.8022 \mathrm{e}-03$ & - \\
2 & 0.05 & 0.05 & $7.8839 \mathrm{e}-04$ & 3.5441 \\
3 & 0.025 & 0.025 & $2.5968 \mathrm{e}-04$ & 3.0360 \\
4 & 0.0125 & 0.0125 & $9.9315 \mathrm{e}-05$ & 2.6147 \\
5 & 0.00625 & 0.00625 & $4.2617 \mathrm{e}-05$ & 2.3304 \\
6 & 0.003125 & 0.003125 & $1.9685 \mathrm{e}-05$ & 2.1649 \\
\hline
\end{tabular}

with $\Omega$ being the unit square and

$$
b(u)= \begin{cases}u^{1 / m}-u / m & \text { for } u \in[0,1] \\ 1-1 / m & \text { for } u>1\end{cases}
$$

We have now the slow diffusion case at $\bar{u}=0$ and fast diffusion case at $\bar{u}=1$. The assumption (A2) is fulfilled again with $\alpha=1 / m$. The source term and appropriate initial and boundary conditions are considered in order to have an explicit solution of (5.3) given by

$$
u(t, x, y)=24 \operatorname{tx}(1-x) y(1-y)+\epsilon
$$

with $\epsilon$ denoting a small regularization parameter. This parameter has been introduced because the fully discrete nonlinear problem arising are solved by the Newton method. In particular, this choice guarantees the convergence of the Newton method (see [27]). Notice that by introducing the regularization parameter $\varepsilon$, the solution $u$ is bounded away from 0 . A numerical scheme exploiting this idea of perturbing the data for preventing the solution to reach the degeneracy values is analyzed in [24]. We mention here that the elliptic case was also treated in [28]. For the above problem, the final computational time is $T=1$ and $m=2$. We took $\varepsilon=10^{-14}$. Table 5 presents the results obtained for $\tau=h$, and both being halved successively. Clearly, the computations show a convergence order of $h$. Further, we also performed computations for $\tau=h^{1.2}, h$ being again halved successively. According to the estimates of 
Table 6 Numerical results (final time $\mathrm{T}=1, m=2$ )

\begin{tabular}{lllll}
\hline $\mathrm{N}$ & $\tau$ & $\mathrm{h}$ & Error & Reduction \\
\hline 1 & 0.0625 & 0.1 & $2.536576 \mathrm{e}-03$ & - \\
2 & 0.0278 & 0.05 & $6.703072 \mathrm{e}-04$ & 3.7842 \\
3 & 0.012 & 0.025 & $1.925544 \mathrm{e}-04$ & 3.4811 \\
4 & 0.0052 & 0.0125 & $6.034787 \mathrm{e}-05$ & 3.1907 \\
5 & 0.0023 & 0.00625 & $2.087281 \mathrm{e}-05$ & 2.8912 \\
\hline
\end{tabular}

Theorem 4.8, the convergence should be improved to an order of at least $h^{1.2}$. The results presented in Table 6 are confirming the expectations again.

\section{Conclusions}

We have analyzed a numerical scheme for a class of degenerate parabolic equations, including both slow and fast diffusion cases. In particular, the results apply to the Richards equation modeling the flow in unsaturated porous media. The spatial discretization is mixed and based on the lowest order Raviart-Thomas finite elements, whereas the time stepping is performed by the Euler implicit method. We have proven the convergence of the scheme by estimating the error in terms of the discretization parameters. The numerical experiments agree with the estimates derived theoretically.

Acknowledgements The work of the second author has been supported by the Dutch BSIK/BRICKS MSV-1 project. We thank the anonymous referees for their questions and remarks that helped us to improve the paper.

\section{References}

1. Aavatsmark, I.: An introduction to multipoint flux approximations for quadrilateral grids. Comput. Geosci. 6, 404-432 (2002)

2. Adams, R.A.: Sobolev Spaces. Academic Press, New York (1975)

3. Alt, H.W., Luckhaus, S.: Quasilinear elliptic-parabolic differential equations. Math. Z. 183, 311341 (1983)

4. Arbogast, T., Wheeler, M.F., Zhang, N.Y.: A nonlinear mixed finite element method for a degenerate parabolic equation arising in flow in porous media. SIAM J. Numer. Anal. 33, 1669-1687 (1996)

5. Baranger, J., Maitre, J.F., Oudin, F.: Connection between finite volume and mixed finite element methods. M2N Math. Model. Numer. Anal. 30, 445-465 (1996)

6. Barenblatt, G.I.: On some unsteady motion of a liquid or a gas in a porous medium. Prikl. Math. Meh. 16, 67-78 (1952)

7. Barrett, J.W., Knabner, P.: Finite Element Approximation of The Transport of Reactive Solutes in Porous Media. Part II: Error Estimates for Equilibrium Adsorption Processes. SIAM J. Numer. Anal. 34, 455-479 (1997)

8. Bastian, P., Birken, K., Johanssen, K., Lang, S., Neuss, N., Rentz-Reichert, H., Wieners, C.: UG-a flexible toolbox for solving partial differential equations. Comput. Visualiz. Sci. 1, 27-40 (1997)

9. Bause, M., Knabner, P.: Computation of variably saturated subsurface flow by adaptive mixed hybrid finite element methods. Adv. Water Res. 27, 565-581 (2004)

10. Bear, J., Bachmat, Y.: Introduction to Modelling of Transport Phenomena in Porous Media. Kluwer Academic, Dordrecht (1991) 
11. Brezzi, F., Fortin, M.: Mixed and Hybrid Finite Element Methods. Springer, New York (1991)

12. Ciarlet, P.G.: The Finite Element Method for Elliptic Problems. North-Holland, Amsterdam (1978)

13. Van Duijn, C.J., Knabner, P.: Solute transport in porous media with equilibrium and nonequilibrium multiple-site adsorption: travelling waves. J. Reine Angew. Math. 415, 1-49 (1991)

14. Ebmeyer, C.: Error estimates for a class of degenerate parabolic equations. SIAM J. Numer. Anal. 35, 1095-1112 (1998)

15. Evans, L.C.: Partial Differential Equations. American Mathematical Society, Providence (1998)

16. Eymard, R., Gutnic, M., Hillhorst, D.: The finite volume method for Richards equation. Comput. Geosci. 3, 256-294 (1999)

17. Eymard, R., Hilhorst, D., Vohralík, M.: A combined finite volume-nonconforming/mixed-hybrid finite element scheme for degenerate parabolic problems. Numer. Math. 105, 73-131 (2006)

18. Ivanov, A.V., Jäger, W.: Existence and uniqueness of a regular solution of Cauchy-Dirichlet problem for equation of turbulent filtration. J. Math. Sci. (New York) 101, 3472-3502 (2000)

19. Ladyzhenskaya, O.A., Ural'tseva, N.N.: Linear and Quasilinear Elliptic Equations. Academic Press, London (1968)

20. Lu, Y., Jäger, W.: On solutions to nonlinear reaction-diffusion-convection equations with degenerate diffusion. J. Diff. Eqs. 170, 1-21 (2001)

21. Nochetto, R.H., Verdi, C.: Approximation of degenerate parabolic problems using numerical integration. SIAM J. Numer. Anal. 25, 784-814 (1988)

22. Otto, F.: $L^{1}$-contraction and uniqueness for quasilinear elliptic-parabolic equations. J. Diff. Eqs. 131, 20-38 (1996)

23. Pop, I.S.: Error estimates for a time discretization method for the Richards' equation. Comput. Geosci. 6, 141-160 (2002)

24. Pop, I.S., Yong, W.A.: A numerical approach to degenerate parabolic equations. Numer. Math. 92, 357381 (2002)

25. Quarteroni, A., Valli, A.: Numerical Approximations of Partial Differential Equations. Springer, Heidelberg (1994)

26. Radu, F.A., Pop, I.S., Knabner, P.: Order of convergence estimates for an Euler implicit, mixed finite element discretization of Richards' equation. SIAM J. Numer. Anal. 42, 1452-1478 (2004)

27. Radu, F.A., Pop, I.S., Knabner, P. : On the convergence of the Newton method for the mixed finite element discretization of a class of degenerate parabolic equation. In: Castro Bermudez de, A. et al. (ed.) Numerical Mathematics and Advanced Applications, pp. 1194-1200. Springer, Heidelberg (2006)

28. Radu, F.A., Bause, M., Knabner, P., Friess, W., Metzmacher, I.: Numerical simulation of drug release from collagen matrices by enzymatic degradation. Comput. Visualiz. Sci. (2007, in press)

29. Rulla, J.: Error analysis for implicit approximations to solutions to Cauchy problems. SIAM J. Numer. Anal. 33, 68-87 (1996)

30. Schneid, E., Knabner, P., Radu, F.A.: A priori error estimates for a mixed finite element discretization of the Richards' equation. Numer. Math. 98, 353-370 (2004)

31. Schneid, E.: Hybrid-Gemischte Finite-Elemente-Diskretisierung der Richards-Gleichung (in German). Ph.D. thesis. University of Erlangen-Nürnberg (2000). also available at http://www.am.uni-erlangen. de/am1/publications/dipl_phd_thesis/dipl_phd_thesis.html

32. Schweizer, B.: Regularization of outflow problems in unsaturated porous media with dry regions. J. Diff. Eqs. 237, 278-306 (2007)

33. Thomas, J.M.: Sur l'analyse numérique des méthodes d'éléments finis hybrides et mixtes. Thése d'Etat. Université Pierre \& Marie Curie, Paris 6 (1977)

34. Woodward, C., Dawson, C.: Analysis of expanded mixed finite element methods for a nonlinear parabolic equation modeling flow into variably saturated porous media. SIAM J. Numer. Anal. 37, 701724 (2000)

35. Yotov, I.: A mixed finite element discretization on non-matching multiblock grids for a degenerate parabolic equation arizing in porous media flow. East-West J. Numer. Math. 5, 211-230 (1997) 